\title{
Oscillations with uniquely long periods in a microfluidic bubble generator
}

\author{
PIOTR GARSTECKI ${ }^{1,2 *}$, MICHAEL J. FUERSTMAN ${ }^{1}$ AND GEORGE M. WHITESIDES ${ }^{1 *}$ \\ ${ }^{1}$ Department of Chemistry and Chemical Biology, Harvard University, 12 Oxford St., Cambridge, Massachusetts, USA \\ ${ }^{2}$ Institute of Physical Chemistry, Polish Academy of Sciences, Kasprzaka 44/52, 01-224 Warsaw, Poland \\ *e-mail: garst@ichf.edu.pl; gwhitesides@gmwgroup.harvard.edu
}

U nderstanding spatiotemporal complexity ${ }^{1-3}$ is important to many disciplines, from biology $y^{4,5}$ to finance ${ }^{6}$. However, because it is seldom possible to achieve complete control over the parameters that determine the behaviour of real complex systems, it has been difficult to study such behaviour experimentally. Here we demonstrate a simple microfluidic bubble generator that shows stable oscillatory patterns (both in space and time) of unanticipated complexity and uniquely long repetition periods. At low flow rates, the device produces a regular stream of bubbles of uniform size. As the flow increases, the system shows intricate dynamic behaviour typified by a stable limit cycle of order 29 bubbles per period, which repeats without change over intervals of up to 100 periods and more. As well as providing an example of a well-characterized and experimentally tractable model system with which to study complex, nonlinear dynamics, such behaviour demonstrates that it is possible to observe complex and stable limit cycles without active external control.

Even though nonlinear temporal dynamics is well understood ${ }^{1}$, the understanding of systems having complex dynamics in both time and space is still limited ${ }^{2,3,7}$. Spatiotemporal dynamics may underlie phenomena as varied as weather ${ }^{8}$, evolution of geophysical patterns ${ }^{9}$, the movement of stock markets ${ }^{6}$, the flux through metabolic pathways ${ }^{4}$, biomechanical processes ${ }^{10}$ and morphogenesis ${ }^{2,5}$. So-called large spatiotemporal systemssystems in which the number of effective degrees of freedom is large-demonstrate the applicability of amplitude and phase equations $s^{2,11}$ in the characterization of spatiotemporal patterns $s^{2,7}$. Small systems — systems with only a few degrees of freedom-are substantially more complicated to study because the boundaries of these systems strongly influence their dynamics and are difficult to treat analytically. Truly tractable experimental demonstrations ${ }^{12,13}$ of spatiotemporal dynamics are scarce $^{11}$, and the need for them is correspondingly high ${ }^{2}$. Here we provide an example of such a tractable system that is amenable to rational design and modification.

The system comprises coupled microfluidic flow-focusing devices. A single flow-focusing device ${ }^{14,15}$ (Fig. 1a) was first implemented in a microfluidic chip by Anna et al. ${ }^{16}$; the system has three inlet channels-two outer supplying liquid and the centre one supplying gas - that merge at a junction leading to a single outlet channel. In this geometry, the pinch-off process that generates bubbles is regulated ${ }^{17}$ by the inflow of liquid into the orifice (at a typical speed $u_{\text {inflow }} \sim 0.01-1 \mathrm{~m} \mathrm{~s}^{-1}$ ); this inflow is slower by two to five orders of magnitude than the relaxation rates (the typical interfacial and bulk relaxation speeds are $u_{\text {interfacial }} \sim \gamma / \mu \sim 100 \mathrm{~m} \mathrm{~s}^{-1}$ and $u_{\text {sound }} \sim 1,000 \mathrm{~m} \mathrm{~s}^{-1}$, respectively, where $\gamma$ is the interfacial tension and $\mu$ is the viscosity of the liquid). As a result, the breakup process in the simple flow-focusing device is highly reproducible and leads to the generation of monodisperse bubbles ${ }^{17,18}$.

We have incorporated this well-characterized ${ }^{17}$ device into a multi-orifice design (Fig. 1b) in which the stream of gas can pinch-off at any orifice in an array of five orifices. Each liquid inlet splits into five connected channels that feed the array of orifices. These five channels couple the dynamics at each orifice by pressure feedback loops. For an externally fixed rate of flow, $Q$, of the liquid, the viscous resistance to flow downstream determines the pressure at each orifice and the flow through it. As the gas fills the centre channel, it blocks certain orifices and increases the flow through a smaller number of (unblocked) inlet channels. The pressures $\left(p_{\mathrm{i}}\right)$ in-and the rates of inflow $\left(q_{\mathrm{i}}\right)$ into-each of the orifices depend on the length and position of the gaseous thread and of the bubbles. When the thread breaks and the bubbles move downstream, they also alter the distribution of pressure, and consequently the dynamics of the breaking of the thread.

We examined the behaviour of this system using $\mathrm{N}_{2}$ as the gas and water containing $2 \%$ of a surfactant (Tween 20) as the liquid. As the pressure applied to the stream of gas increases, the behaviour of the system becomes progressively more complex and then it re-simplifies. For a fixed $Q$, and for the pressure applied to the gas stream $p$ only slightly above the minimum pressure needed to form bubbles, the thread breaks into bubbles of uniform size at the first orifice (the 'period-1' regime). At intermediate pressures the dynamics becomes astonishingly complex: the thread enters the array of orifices, undergoes several breakup events that generate a series of bubbles of different sizes, pauses, and then the whole process repeats. In spite of the intricacy of this periodic behaviour, 


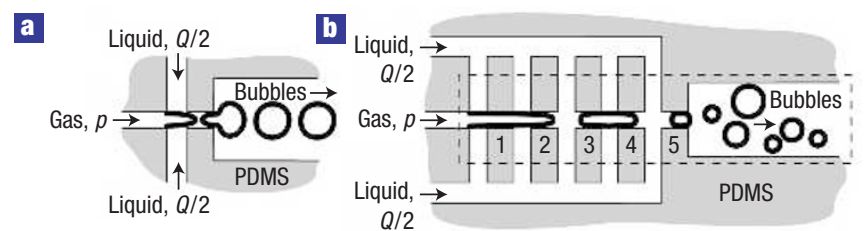

G

d
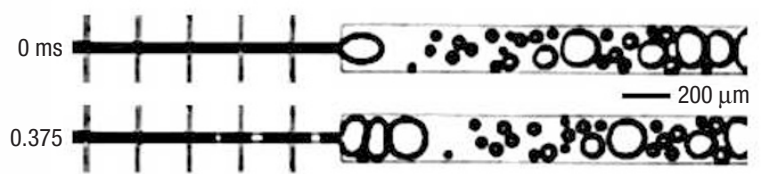

$\mathbf{E}$

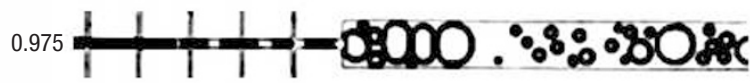

$\mathbf{f}$

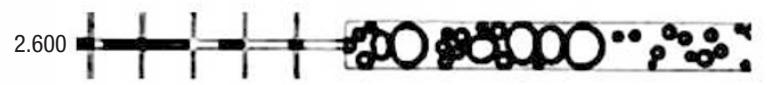

g

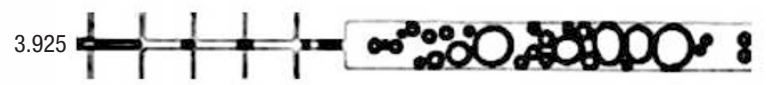

h

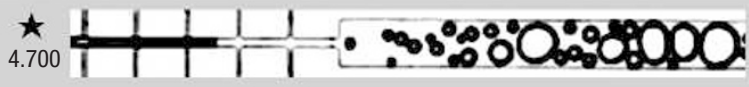

i.

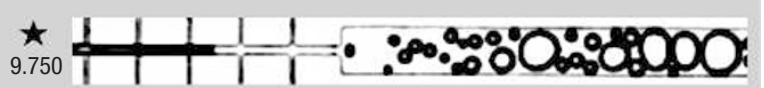

j

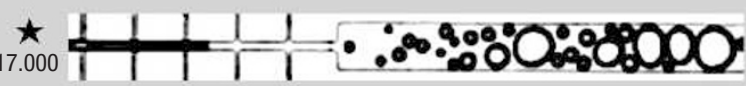

k

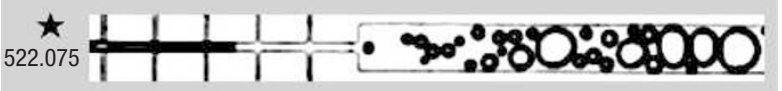

Figure 1 The geometry of the system and an example of the periodic behaviour. a,b, Schematic diagrams of the flow-focusing device with a single orifice (a) and the multi-orifice system (b) that we used in this study. We supply the gaseous phase (nitrogen) through a channel that runs along the axis of the device. Liquid (water containing $2 \% \mathrm{w} / \mathrm{w}$ Tween 20 surfactant) flows from both sides into the central channel through a network of five side channels; the five orifices in which bubbles are formed are numbered from 1 to 5 . The width of the gas-inlet channel and the orifices is $40 \mu \mathrm{m}$, the channels that deliver the liquid to the orifices comprise two sections of different widths, and the width of the section adjacent to the centre-line channel is $20 \mu \mathrm{m}$. The spacing between the liquid-carrying inlets along the centre channel (the lengths of the orifices) and the width of the outlet channel are $200 \mu \mathrm{m}$. All channels have a uniform height of $44 \mu \mathrm{m}$ (the Supplementary Information gives a detailed diagram of the experimental device). The gas-liquid interface is represented with a thick solid line. The shaded areas correspond to the elastomeric polydimethylsiloxane (PDMS) walls of the device. c-k, The dynamics of the system at $Q=1.66 \mu \mathrm{s} \mathrm{s}^{-1}$ and the value of the pressure applied to the gas stream $p=76 \mathrm{kPa}$. The part of the system shown in these micrographs corresponds to the area marked by the dashed box in diagram $\mathbf{b}$. c-h are chronological micrographs of the system showing formation of the sequence of 29 bubbles produced within one period. This sequence is repeated indefinitely provided that the pressures applied to the gas and liquid inlets remain approximately constant. $\mathbf{h}-\mathbf{k}$ (highlighted with the grey background) illustrate the system at the same phase of the period - that is right after the last of the 29 bubbles enters the outlet channel. The intervals between the pairs of micrographs $\mathbf{h}$ and $\mathbf{i}, \mathbf{i}$ and $\mathbf{j}$, and $\mathbf{j}$ and $\mathbf{k}$ correspond to 1,101 and 1 period(s) correspondingly. The numbers by the images shown in c-k indicate the time (in ms) elapsed after the first micrograph (c) was taken.

it is coherent-the sequence of the sizes of the bubbles and the intervals between them are almost identical from period to period (Fig. 1h-k). We have observed stable, coherent sequences of order as high as 40 bubbles in each period. At high values of $p$, the thread progresses all the way downstream and breaks only in the
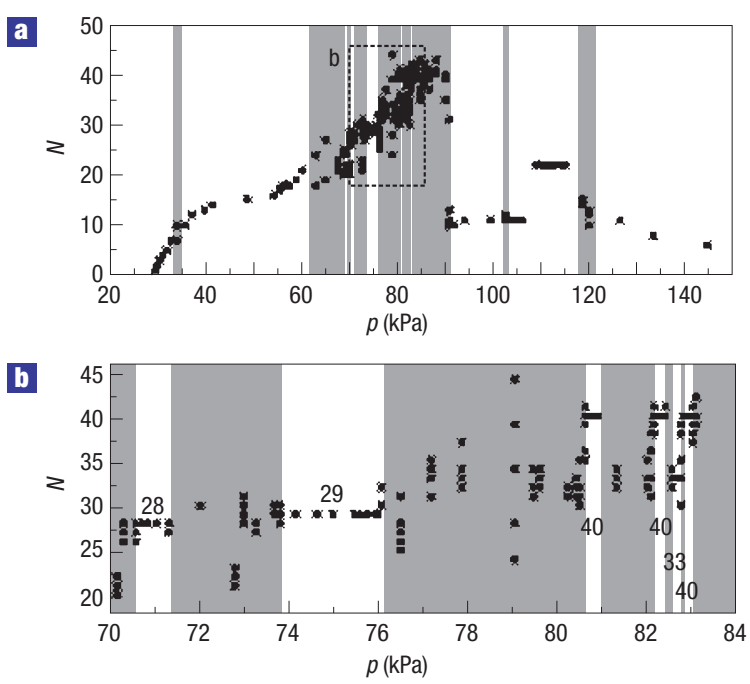

Figure 2 Periodicity as a function of the pressure applied to the stream of gas. a, The number $(N)$ of bubbles in a period (or burst) as a function of $p$, for $Q=1.66 \mu \mathrm{l} \mathrm{s}^{-1}$. The regions in which the cyclic trajectories are stable (white) are separated by regions of irregular dynamics (shaded grey). For each pressure we counted the number of bubbles in five consecutive bursts. If this number was always the same (suggesting a stable cyclic orbit) we have verified that the behaviour does not change over longer intervals (100 periods). $\mathbf{b}$, An enlarged plot of a section of the diagram (marked with the dashed lines in $\mathbf{a}$ ). The numbers in $\mathbf{b}$ denote the order of the limit cycle that is stable within the white (not shaded) band.

last orifice, again in a period-1 mode. We can adjust the periodicity almost continuously from order $1\left(Q=1.66 \mu \mathrm{l} \mathrm{s}^{-1}, p=29.75 \mathrm{kPa}\right)$ to $40\left(Q=1.66 \mu \mathrm{s} \mathrm{s}^{-1}, p=80.80 \mathrm{kPa}\right)$, see Fig. 2. Each cyclic trajectory is stable within a finite range of pressures: the period-29 cycle is stable for $p$ in the range between 74.2 and $76 \mathrm{kPa}$.

To exemplify the complex periodic dynamics we describe the behaviour of the system at $Q=1.66 \mu \mathrm{s} \mathrm{s}^{-1}$ and $p=76 \mathrm{kPa}$. For these values, there are 29 breakup events and 29 bubbles formed in each cycle. Figure $1 \mathrm{c}-\mathrm{h}$ shows six chronological micrographs of the system taken within one period. Micrographs $1 \mathrm{~h}-\mathrm{k}$ depict the system at the same phase in the cycle-when the last of the 29 bubbles emerges from the fifth orifice. This periodic behaviour is stable over long times $\left(10^{3} \mathrm{~s}\right.$, corresponding to $10^{5}$ periods $)$, and we were able to recover the same behaviour of the system in multiple copies of the device.

To quantify the coherence of this behaviour, we construct a function $\rho(t)$, where $t$ is time, that can assume two values: $\rho(t)=1$ if the outlet of the last (fifth) orifice is occupied by gas, and $\rho(t)=-1$ if it is occupied by water. Figure 3 a demonstrates four fragments of $\rho(t)$ extracted from a video tracing the behaviour of the system over $4.3 \mathrm{~s}$ with a temporal resolution of $6.25 \mu \mathrm{s}$. Each change of $\rho(t)$ from -1 to 1 signifies an approach of the gas phase to the end of the last orifice; a release of a bubble into the outlet channel changes $\rho(t)$ from 1 to -1 . Figure $3 \mathrm{~b}$ shows the autocorrelation function $g(\Delta t)=\langle\rho(t) \rho(t+\Delta t)\rangle$, where \langle\rangle denotes an average over the 690,000 frames $(4.31 \mathrm{~s})$, with peaks at the multiples of $\Delta t_{29}=5.02 \mathrm{~ms}$ - an interval corresponding to a sequence of 29 bubbles.

We verify the stability of these periodic dynamics by constructing Poincaré maps ${ }^{1}$ on the intervals $\tau_{n}$ between adjacent bubbles. A release of the $n$th bubble is signified by the $n$th instance of a change of $\rho(t)$ from 1 to -1 at $t \equiv t_{n}$. We define the $n$th interval as $\tau_{n}=t_{n}-t_{n-1}$. Figure $3 \mathrm{c}$ shows a map of $\tau_{n}$ versus $\tau_{n-1}$ plotted for 29 consecutive intervals; Fig. 3d shows 
a

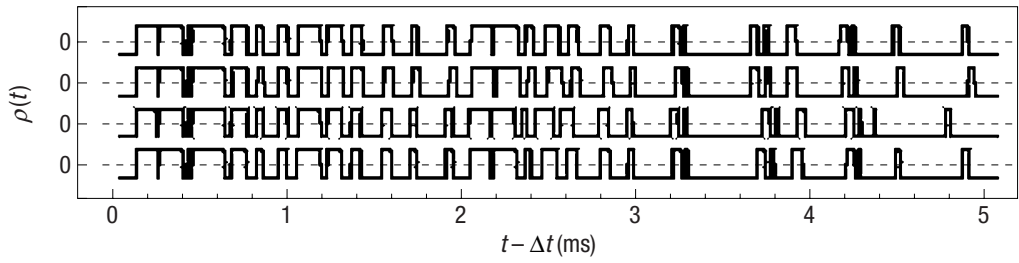

b

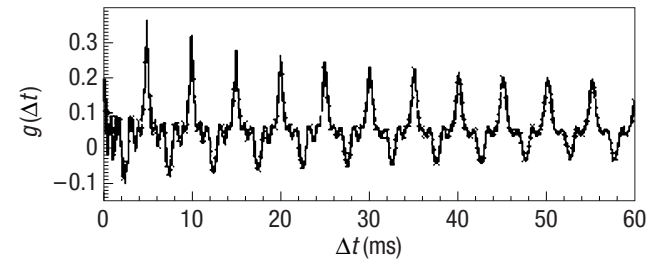

f

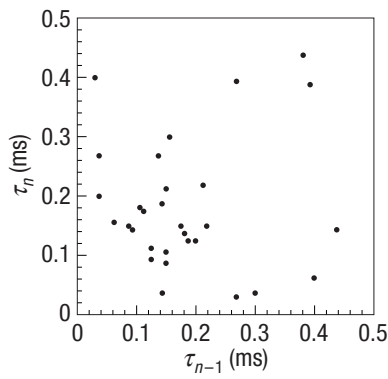

d

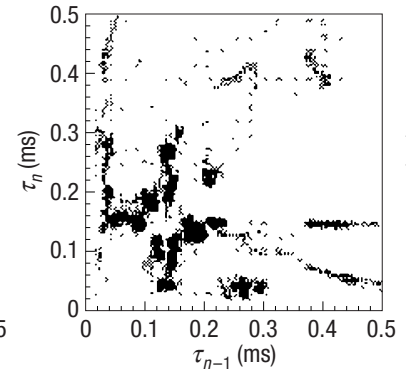

Q

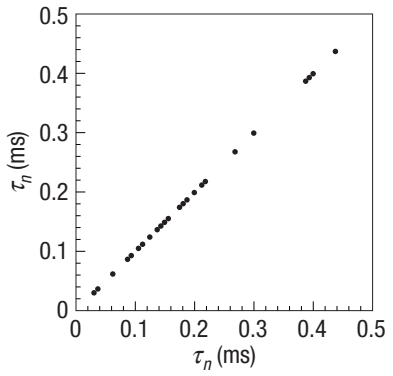

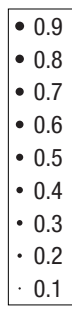

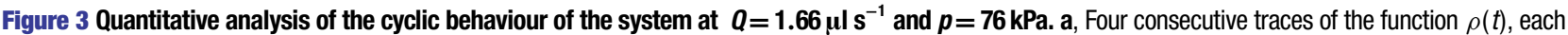
representing one period; the second, third and fourth trace are delayed by $\Delta t=5.037,10.112$ and $15.100 \mathrm{~ms}$, respectively. One can see that a small deviation in the intervals between bubbles (third trace) does not amplify, but decays to yield nearly ideal behaviour in the following period (fourth trace). $\mathbf{b}$, The autocorrelation function $g(\Delta t)$ calculated for the full trace of $\rho(t)$ (interval of $4.3215 \mathrm{~s}$ ). The peaks occur at multiples of $\Delta t_{29}=5.020 \mathrm{~ms}$. c,d, The Poincaré maps of the intervals $\tau_{n}\left(\tau_{n-1}\right)$ between bubbles plotted for one period (c), and for the whole series of data of 25,257 bubbles (d). e,f, The Poincaré maps for $\tau_{n}\left(\tau_{n-29}\right)$, again, plotted for a single period (e) and for the whole series of data (f). As 29 is a prime number, the concentration of points on the diagonal cannot be explained by a resonance with a lower-order period. The size of the spots on the Poincare maps $\mathbf{d}$ and $\mathbf{f}$ code the number of counts for each point. We have normalized the counts by dividing them by the largest count $\left(c_{i j}=c_{i j} / c_{\max }\right.$, where $c_{i j}$ is the number of counts for each point, and $c_{\max }$ is the maximum number of counts). In order to bring out the intermediate values of counts we used a nonlinear scaling of the diameter $(d)$ of the dots: $d$ proportional to $1+(3 / 4) \log \left(c_{i j}\right)$. This scale is shown to the right of panel $\mathbf{f}$.

the same map for all the 25,257 intervals extracted from the $4.31 \mathrm{~s}$ recording. Strong clustering of the points signifies periodic dynamics, and the positions of the clusters match the locations of the points on the map for a single period. We also plot a map of $\tau_{n}$ versus $\tau_{n-29}$ in Fig. 3f. If a system showed ideally periodic behaviour, all of the points on the plot would lie on the line $\tau_{n}=\tau_{n-29}$. As 29 is a prime number, the observed concentration of points on the diagonal cannot be explained by a resonance with a lower period (as would be possible in the case for a $\tau_{n}$ versus $\tau_{n-16}$ map of a system showing period-4 behaviour). We do not observe such clustering for any map of $\tau_{n}$ versus $\tau_{n-m}$ with $m<29$.

There are two causes of the scatter of points in the plots shown in Fig. 3d and f. First, some bubbles flow through the last orifice very rapidly and our ability to detect them is at the limits of the temporal resolution of our camera $(6.25 \mu \mathrm{s})$. Some of the bubbles are omitted, and these omissions introduce errors into the sequence of intervals $\left(\tau_{n}\right)$. Second, within some periods, the intervals between consecutive bubbles vary slightly (see the third trace in Fig. 3a). The observation that these variations do not propagate into irregular or chaotic behaviour indicates that the observed periodic trajectories are stable limit cycles and small deviations from them decay rather than amplify with time. This stability is in pronounced contrast with other nonlinear systems ${ }^{1,19}$ in which the multiperiodic trajectories are usually intertwined in strange attractors ${ }^{20}$, are unstable, and the observed behaviour of the system is irregular.

We believe that the stability of the long limit cycles reflects the separation of timescales between the two sets of processes that determine the behaviour of the system: (i) slow breakup of the gaseous thread, which typically proceeds at a timescale $t_{\text {breakup }} \sim 10 \mu \mathrm{m} / u_{\text {inflow }} \sim 100 \mu \mathrm{s}$, with $10 \mu \mathrm{m}$ ( radius of the thread) as the characteristic length scale for the interfacial dynamics; and (ii) fast spatial coupling between different positions along the breaking thread, which proceeds at a typical timescale $t_{\text {coupling }} \sim 1 \mathrm{~mm} / u_{\text {sound }} \sim 1 \mu \mathrm{s}$, where $1 \mathrm{~mm}$ is a typical length of the channels that transmit the feedback in pressure. This separation insulates the system against perturbations, and leads to stable periodic behaviour. The construction of our system out of modules (single flow-focusing devices) characterized by 'linear' dynamics ${ }^{17}$, resulting from the separation of timescales between the slow evolution of the system and fast relaxation rates, distinguishes the multi-orifice device from several other hydrodynamic systems that show archetypal examples of nonlinear dynamics, for example, the famous leaky faucet system ${ }^{21,22}$ or spatially extended dripping systems ${ }^{13}$.

Until now, interest in the field of spatiotemporal dynamics has focused more on probing the onset and properties of chaos, and less on selecting and understanding intricate periodic behaviour. An exception is the work of Ott et al. ${ }^{23}$, who demonstrated active stabilization of an unstable periodic cycle embedded in a strange attractor. Their method ${ }^{23}$ required continuous application of judiciously chosen perturbations to keep the system on a periodic (but unstable) trajectory. Similar strategies have stabilized periodic dynamics in simple mechanical ${ }^{24}$, electronic ${ }^{25}$ and biological systems $^{26}$. Ott et al. ${ }^{23}$ argued that the ability to choose and stabilize periodic trajectories may lead to the construction of adaptive materials and shed light on the adaptability and selfregulation shown in life ${ }^{10}$. The intricate nonlinear phenomena, especially those in the world of biology, such as, for example, morphogenesis ${ }^{5}$, dynamics of populations ${ }^{20,27,28}$ or the operation of the biological machinery regulating the distribution of enzymes in cells $s^{29}$, often involve intricate correlations in both space and time that are only possible in strongly nonlinear systems that operate at the edge of chaos $^{30}$. The proximity to chaotic dynamics 
induces sensitivity to perturbations, which destroys the temporal and spatial correlations; this feature makes the experimental studies of the complex spatiotemporal patterns difficult. The example of a system that we demonstrated here-a system that shows complex yet stable periodic dynamics without active external controlsuggests that the separation of timescales of the (slow) dynamics and the (fast) spatial coupling could serve as a guideline in synthesis of artificial systems showing complex nonlinear behaviour and possibly help in the studies of spatiotemporal dynamics. In a shorter perspective, modifications of the system presented here could open a way to preparation of emulsions characterized by a multimodal, space-filling distribution of sizes of the bubbles or droplets. The example of a coherent periodic behaviour in this microfluidic systems points to the potential for design of lab-onchip devices that would reliably and reproducibly perform simple signal-processing tasks relying on nonlinear dynamics.

Received 7 July 2005; accepted 14 October 2005; published 1 December 2005.

References

1. Schuster, H. G. Deterministic Chaos: An Introduction (Wiley-VCH, Weinheim, 2003).

2. Cross, M. C. \& Hohenberg, P. C. Pattern-formation outside of equilibrium. Rev. Mod. Phys. 65, 851-1112 (1993).

3. Aranson, I. S. \& Kramer, L. The world of the complex Ginzburg-Landau equation. Rev. Mod. Phys. 74, 99-143 (2002)

4. Stelling, J., Klamt, S., Bettenbrock, K., Schuster, S. \& Gilles, E. D. Metabolic network structure determines key aspects of functionality and regulation. Nature 420, 190-193 (2002).

5. Turing, A. M. The chemical basis of morphogenesis. Phil. Trans. R. Soc. Lond. B 237, 37-72 (1952)

6. Gabaix, X., Gopikrishnan, P., Plerou, V. \& Stanley, H. E. A theory of power-law distributions in financial market fluctuations. Nature 423, 267-270 (2003).

7. Gollub, J. P. \& Cross, M. C. Nonlinear dynamics: Chaos in space and time. Nature 404, 710-711 (2000)

8. Nicolis, C. \& Nicolis, G. Is there a climatic attractor? Nature 311, 529-532 (1984).

9. Bak, P., Tang, C. \& Wiesenfeld, K. Self-organized criticality—an explanation of 1/F noise. Phys. Rev. Lett. 59, 381-384 (1987).

10. Glass, L. Synchronization and rhythmic processes in physiology. Nature 410, 277-284 (2001).

11. Cladis, P. E. \& Palffy-Muhoray, P. Spatio-Temporal Patterns in Nonequilibrium Complex Systems (Addison-Wesley, Reading, Massachusetts, 1994).

12. Ecke, R. E., Hu, Y. C., Mainieri, R. \& Ahlers, G. Excitation of spirals and chiral-symmetry breaking in Rayleigh-Benard convection. Science 269, 1704-1707 (1995).
13. Brunet, P. \& Limat, L. Defects and spatiotemporal disorder in a pattern of falling liquid columns. Phys. Rev. E 70, 046207 (2004)

14. Ganan-Calvo, A. M. Generation of steady liquid microthreads and micron-sized monodisperse sprays in gas streams. Phys. Rev. Lett. 80, 285-288 (1998).

15. Ganan-Calvo, A. M. \& Gordillo, J. M. Perfectly monodisperse microbubbling by capillary flow focusing. Phys. Rev. Lett. 87, 274501 (2001).

16. Anna, S. L., Bontoux, N. \& Stone, H. A. Formation of dispersions using 'flow focusing' in microchannels. Appl. Phys. Lett. 82, 364-366 (2003).

17. Garstecki, P., Stone, H. A. \& Whitesides, G. M. Mechanism for flow-rate controlled breakup in confined geometries: A route to monodisperse emulsions. Phys. Rev. Lett. 94, 164501 (2005).

18. Garstecki, P. et al. Formation of monodisperse bubbles in a microfluidic flow-focusing device. Appl. Phys. Lett. 85, 2649-2651 (2004).

19. Feigenbaum, M. J. Quantitative universality for a class of non-linear transformations. J. Stat. Phys. 19, 25-52 (1978).

20. King, A. A. et al. Anatomy of a chaotic attractor: Subtle model-predicted patterns revealed in population data. Proc. Natl Acad. Sci. USA 101, 408-413 (2004).

21. Ambravaneswaran, B., Phillips, S. D. \& Basaran, O. A. Theoretical analysis of a dripping faucet. Phys. Rev. Lett. 85, 5332-5335 (2000)

22. Coullet, P., Mahadevan, L. \& Riera, C. Hydrodynamical models for the chaotic dripping faucet. J. Fluid Mech. 526, 1-17 (2005)

23. Ott, E., Grebogi, C. \& Yorke, J. A. Controlling chaos. Phys. Rev. Lett. 64, 1196-1199 (1990).

24. Ditto, W. L., Rauseo, S. N. \& Spano, M. L. Experimental control of chaos. Phys. Rev. Lett. 65 , 3211-3214 (1990)

25. Hunt, E. R. Stabilizing high-period orbits in a chaotic system-the diode resonator. Phys. Rev. Lett. 67, 1953-1955 (1991)

26. Garfinkel, A., Spano, M. L., Ditto, W. L. \& Weiss, J. N. Controlling cardiac chaos. Science 257, 1230-1235 (1992).

27. Hudson, P. J. \& Bjørnstad, O. N. Vole stranglers and lemming cycles. Science 302, 797-798 (2003)

28. Gilg, O., Hanski, I. \& Sittler, B. Cyclic dynamics in a simple vertebrate predator-prey community. Science 302, 866-868 (2003).

29. Goldbeter, A. Computational approaches to cellular rhythms. Nature 420, 238-245 (2002).

30. Langton, C. Computation at the edge of chaos: Phase transitions and emergent computation. Physica D 42, 12-47 (1990).

\section{Acknowledgements}

We would like to thank L. Mahadevan (Harvard University) for helpful discussions. P.G. acknowledges a postdoctoral fellowship from the Foundation for Polish Science. We thank the Harvard MRSEC for the use of high-speed cameras and microfabrication facilities. This work was supported by the US Department of Energy (DE-FG02-00ER45852).

Correspondence and requests for materials should be addressed to P.G. or G.M.W.

Supplementary Information accompanies this paper on www.nature.com/naturephysics.

Competing financial interests

The authors declare that they have no competing financial interests.

Reprints and permission information is available online at http://npg.nature.com/reprintsandpermissions/ 